\title{
Comparación en el enfoque de aprendizaje entre alumnos de grado y máster
}

Leiva-Brondo, M. ${ }^{\text {a }}$, Pérez-de-Castro, A. ${ }^{\mathrm{b}}$

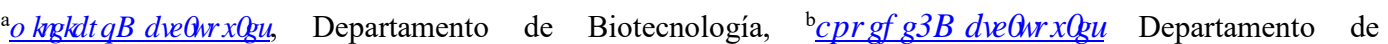
Biotecnología, Universitat Politècnica de València.

\section{\$EWWDWW}

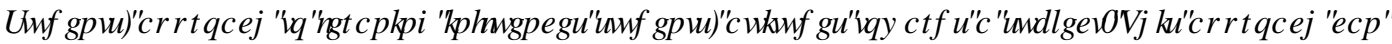

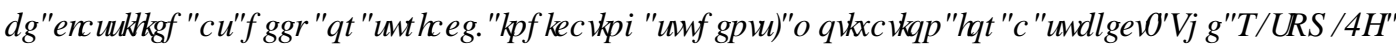

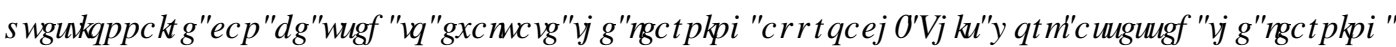
DSS LRDFK LQ WXGHWW LQ D IILWM HDU\%

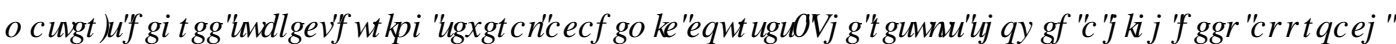

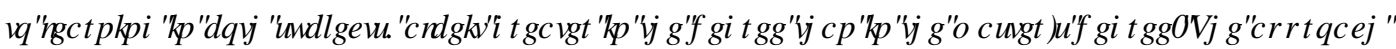

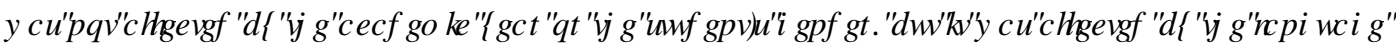

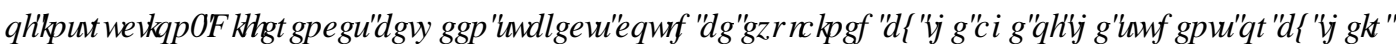

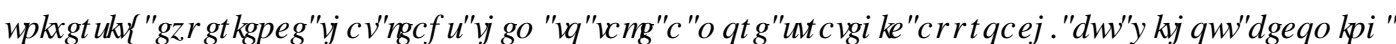
XSHIIFWOI

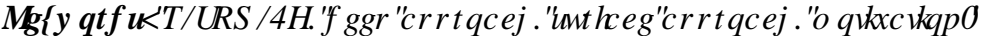

]

\section{HXP HQ}

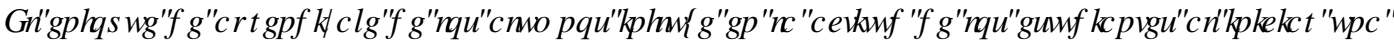

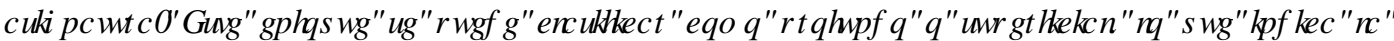

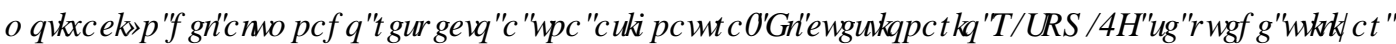

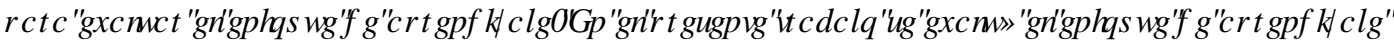

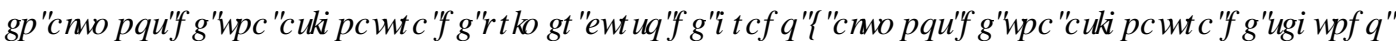

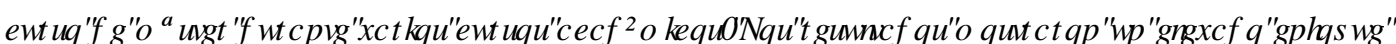

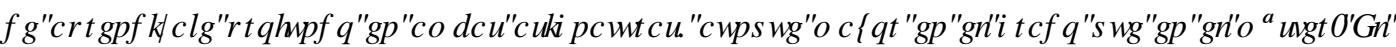

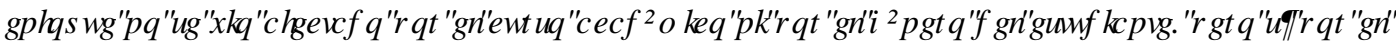

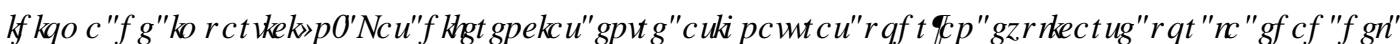

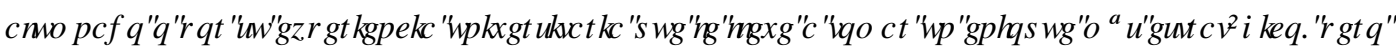
ИQCON DUDUHUXSHUIFIDO]

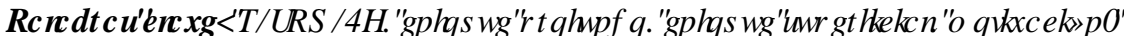

\section{Introducción}

Los alumnos son capaces de adoptar diversos enfoques de aprendizaje (Marton and Säljö, 1976b, 1976a; Graham, Entwistle and Ramsden, 1984; Biggs, 1987). Se han identificado dos enfoques principales de aprendizaje: profundo y superficial, aunque otros autores también identifican un enfoque estratégico (Marton and Säljö, 1976b; Biggs, 1987; Entwistle and Entwistle, 2003). El enfque profundo supone una implicación intrínseca del estudiante en su aprendizaje y se basa en una reflexión sobre el significado de su aprendizaje y un estudio basado en la comprensión (Biggs, 1987; Justicia $H W D O$, 2008; Fryer HMDO, 2012; Frăsineanu, 2013). Por otro lado, el aprendizaje superficial supone que el alumnado está más preocupado 
por las calificaciones que por el aprendizaje y sus esfuerzos se enfocan en superar la asignatura sin una motivación intríseca (Justicia HWDO, 2008; Fryer HWDO, 2012; Frăsineanu, 2013).

Existen varios factores que modifican el enfoque de aprendizaje, ya que no es una característica fija del alumno o alumna (Biggs, 1987; Baeten $H M D O$, 2010). Entre los factores personales se puede incluir la personalidad, pero también la edad, el género o los condicionantes socioculturales (Gijbels HWDQ, 2005; Salamonson $H W D O$, 2013). La edad es un factor que influye y, en general, a mayor edad, mayor enfoque profundo (Gow and Kember, 1990; Richardson and King, 1998; Gijbels HWDO, 2005; Richardson, 2013), aunque también a medida que se avanza en los estudios universitarios el enfoque superficial aumenta (Leiva-Brondo, Cebolla-Cornejo, Peiró, Andrés-Colas, $H M D O$, 2020). Es posible que el alumnado opte por un enfoque más estratégico en detrimento del enfoque profundo y que aumente el enfoque superficial debido a una adaptación a los sistemas de evaluación que se utilicen (Biggs, 1987; Zeegers, 2001; LeivaBrondo, Cebolla-Cornejo, Peiró, Andrés-Colas, $H \mathbb{W D O}$, 2020). En general, no hay diferencias en función del género del alumnado, (Zeegers, 2001; May HMDQ 2012); en ciertos casos se han encontrado diferencias, aunque ligadas a la edad o factores culturales (Severiens and Dam, 1998; Rubin $H W D O$, 2018). El contexto cultural también es un factor que influye, habiéndose hallado diferencias entre diversos contextos geográficos (Biggs, Kember and Leung, 2001; Justicia HMDO, 2008; Immekus and Imbrie, 2010). También es posible encontrar factores situacionales, a los que el alumnado adapta su enfoque de aprendizaje, como son el tipo de estudio, la asignatura, las actividades en el aula o el sistema de evaluación, y que modifican el enfoque del aprendizaje del alumno (Biggs and Tang, 2007; Rubin HWDO, 2018).

Para evaluar el enfoque de aprendizaje se pueden utilizar diversos cuestionarios como: Study Attitudes and Methods Revised Short Form (SAMS Short Form) (Michael, Michael and Zimmerman, 1985), Revised Approaches to Studying Inventory (RASI) (Entwistle and Tait, 1995) modificado a Approaches and Study Skills Inventory for Students (ASSIST) (Tait, Entwistle and McCune, 1998; Entwistle and Hilary, 2013), Inventory of Learning Process-Revised (ILP-R) (Schmeck, Ribich and Ramanaiah, 1977), Approaches to Learning and Studying Inventory (ALSI) (Entwistle, McCune and Hounsell, 2002), Learning and Study Inventory Strategies (LASSI) (Weinstein, 1987), or Inventory of Learning Styles (ILS) (Vermunt, 1994). El Study Process Questionnaire (SPQ) (Biggs, 1987), que fue posteriormente revisado (R-SPQ-2F) (Biggs, Kember and Leung, 2001), ha sido validado en diversos estudios y condiciones (Justicia HMDO, 2008; Immekus and Imbrie, 2010; Socha and Sigler, 2014).

Para evaluar el enfoque de aprendizaje el cuestionario R-SPQ-2F contiene 20 preguntas con una escala Likert de 5 puntos (Biggs, Kember and Leung, 2001). Cada una de las preguntas se asocia a un enfoque de aprendizaje (profundo DA o superficial SA), que a su vez se divide en dos subescalas estratégica (DS y SS) y motivacional (DM y SM), y que pretende estimar el comportamiento del alumnado en un contexto específico de aprendizaje.

El cuestionario R-SPQ-2F obtenido por Biggs y colaboradores en Hong Kong (Biggs, Kember and Leung, 2001) ha sido evaluado por su consistencia interna en diversos contextos académicos y distintos idiomas (Immekus and Imbrie, 2010; Fryer HWDO, 2012; Merino Soto and Kumar Pradhan, 2013; Stes, de Maeyer and Van Petegem, 2013; Socha and Sigler, 2014; Xie, 2014; Shaik HWDO, 2017; López-Aguado and Gutiérrez-Provecho, 2018; Zakariya, 2019; Jiyed HWDO, 2020), habiéndose observado una estructura de dos factores principales (profundo y superficial), pero no una consistencia para las escalas secundarias. Análisis confirmatorios mostraron un buen ajuste para esta estructura, si bien diversos modelos son posibles (Merino Soto and Kumar Pradhan, 2013; López-Aguado and Gutiérrez-Provecho, 2018; Zakariya HWDO, 2020).

Se han realizado previamente estudios en asignaturas relacionadas con ciencias de la vida (Leiva-Brondo HWDO, 2018a, 2018b, 2019, 2021; Leiva-Brondo, Cebolla-Cornejo, Peiró and Pérez-de-Castro, 2020), pero son necesarios más comparaciones y datos para evaluar diversos factores que puedan afectar al enfoque de 
aprendizaje del alumnado. En el presente estudio se hace una comparación en el enfoque de aprendizaje de alumnos y alumnas de primer año de grado comparados con alumnos y alumnas de segundo año de máster de asignaturas relacionadas, durante varios curso académicos. Se analiza la implicación de los resultados.

\section{Objetivos}

Se pretende evaluar el enfoque de aprendizaje del alumnado de una asignatura de grado y otra de máster mediante el cuestionario R-SPQ-2F durante varios cursos académicos. Además se evaluará la fiabilidad del cuestionario en el contexto académico, estudiando las diferencias en enfoque de aprendizaje en función del curso académico, idioma de impartición, género y nivel de los esudios, tanto para las escalas principales como las secundiarias del cuestionario.

\section{Desarrollo de la innovación}

Los alumnos y alumnas participantes en el estudio pertenecían a las titulaciones del Grado en Biotecnología y del Máster en Mejora Genética Vegetal que se imparte en la Universitat Politècnica de València (UPV). En el grado el alumnado cursaba la asignatura "Genética General" de primer curso con una carga lectiva de 6 ECTS (European credit transfer system, 60 horas lectivas) organizada en 4 ECTS de teoría de aula y prácticas de aula y 2 ECTS de prácticas de laboratorio. La asignatura "Mejora Genética del Rendimiento en los Sistemas Agrarios" de segundo curso, fue la asignatura del máster incluida en el estudio, con una carga lectiva de 5 ECTS (50 horas lectivas) organizados en 4 ECTS de teoría de aula y 1 ECTS de prácticas de informática. El estudio se realizó durante tres cursos académicos consecutivos (2018-19, 2019-20 y 2020-21) para la asignatura de grado (127, 115 y 107 alumnos/as matriculados, respectivamente) y durante cuatro cursos académicos consecutivos (2017-18, 2018-19, 2019-20 y 2020-21) para la asignatura de máster $(13,17,18$ y 18 alumnos/as matriculados, respectivamente).

El cuestionario R-SPQ-2F estaba disponible a través de la plataforma de aprendizaje PoliformaT (basada en Sakai) al inicio de la asignatura y se les informó de la finalidad del estudios. El estudio fue voluntario y no tenía relación con ningún acto de evaluación de la asignatura. La versión del cuestionario utilizada fue una traducción realizada por Muñoz San Roque y colaboradores (Muñoz San Roque, Prieto Navarro and Torre Puente, 2012). Los programas Microsoft $(C$ Excel y Statgraphics centurión XVII (Statpoint Technologies, Inc.) se utilizaron para calcular los valores medios de las diferentes escalas, las correlaciones entre éstos y los valores alfa de Cronbach de fiabilidad del cuestionario.

\section{Resultados y discusión}

La participación del alumnado fue muy elevada (mayor del 80\%) en las dos asignaturas implicadas en el estudio (Tabla 1). Los valores de enfoque profundo fueron superiores al enfoque superficial en ambas asignaturas, todos los cursos académicos y también en función de idioma o género. Trece alumnos ( 8 en grado y 5 en máster, un 3.9\%; datos no mostrados) tuvieron un enfoque sprofundo mayor que superficial. Un enfoque profundo mayor que superficial también se ha observado en otros estudios (Leung, Ginns and Kember, 2008; Emilia, Bloomfield and Rotem, 2012; Mogre and Amalba, 2014; Jeong HWDO, 2019; LeivaBrondo, Cebolla-Cornejo, Peiró, Andrés-Colas, $H W D O$, 2020). Los alumnos de grado mostraron un mayor enfoque profundo que los alumnos de máster y, aunque la edad es un factor que influye en el enfoque de aprendizaje (Zeegers, 2001; Richardson, 2013; Rubin HWDO, 2018), en este caso se puede relacionar también con el hecho de que la motivación es mayor al inicio de sus estudios que en cursos más avanzados. De hecho otros estudios han observado un declive en el enfoque profundo a medida que avanzan los estudios (Leiva-Brondo, Cebolla-Cornejo, Peiró, Andrés-Colas, $H M D O$, 2020). No se encontraron diferencias entre cursos, pero sí entre idioma y en género, aunque solo para SA. El idioma de impartición de la asignatura se ha descrito como un factor que influye en el enfoque de aprendizaje de los alumnos (Bobe and Cooper,

(c)) BY-NC-ND 2021, Universitat Politècnica de València

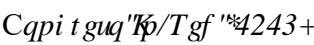


2017; Dong $H M D O$, 2019). En este caso las diferencias pueden ser atribuidas a la diferencia en la nota de acceso, ya que el grupo en inglés tiene una mayor nota de admisión y un menor número de plazas. En general no se encontraron diferencias significativas entre género respecto al enfoque de aprendizaje, excepto en SA. En otros estudios se han identificado diferencias entre géneros relacionadas con la edad del estudiante (Richardson, 2013; Jeong HWDO, 2019). Respecto a las subescalas se observaron resultados similares a los obtenidos con las escalas principales (Tabla 2).

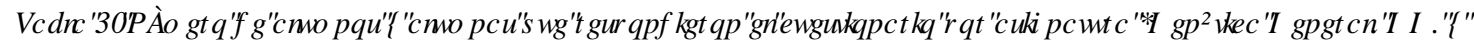

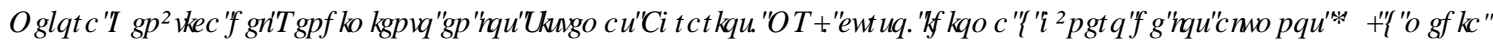

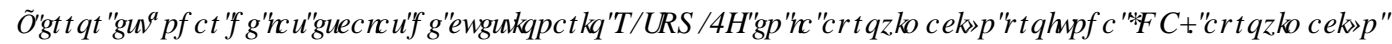

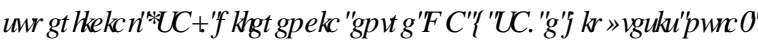

\begin{tabular}{|c|c|c|c|c|c|c|c|c|}
\hline & $\begin{array}{l}\mathrm{N}^{0} \text { respuestas } \\
(\% \text { alumnos } \\
\text { matriculados) }\end{array}$ & DA & & SA & & \multicolumn{2}{|c|}{ Diferencia DA-SA } & $\begin{array}{c}\text { Hipótesis nula } \\
\text { DA-SA }^{2}\end{array}$ \\
\hline \multicolumn{9}{|l|}{ Asignatura } \\
\hline GG & $278(0,80)$ & $3,36 \pm 0,03$ & $\mathrm{~b}^{1}$ & $1,86 \pm 0,03$ & $\mathrm{a}$ & \multicolumn{2}{|l|}{$1,50 \pm 0,05$} & $\mathrm{~b}$ \\
\hline MR & $56(0,85)$ & $3,09 \pm 0,07$ & $\mathrm{a}$ & $2,10 \pm 0,07$ & $\mathrm{~b}$ & \multicolumn{2}{|l|}{$0,99 \pm 0,11$} & $\mathrm{a}$ \\
\hline \multicolumn{9}{|l|}{ Curso } \\
\hline GG 2018-19 & $84(0,66)$ & $3,29 \pm 0,06$ & $\mathrm{a}$ & $1,97 \pm 0,05$ & $\mathrm{~b}$ & $1,32 \pm 0,08$ & $\mathrm{a}$ & $* * *$ \\
\hline GG 2019-20 & $95(0,83)$ & $3,37 \pm 0,06$ & $\mathrm{a}$ & $1,84 \pm 0,04$ & $\mathrm{a}$ & $1,54 \pm 0,08$ & $\mathrm{ab}$ & $* * *$ \\
\hline GG 2020-21 & $99(0,93)$ & $3,41 \pm 0,05$ & $\mathrm{a}$ & $1,79 \pm 0,04$ & $\mathrm{a}$ & $1,62 \pm 0,07$ & $\mathrm{~b}$ & $* * *$ \\
\hline MR 2017-18 & $11(0,85)$ & $3,20 \pm 0,20$ & $\mathrm{a}$ & $1,77 \pm 0,14$ & $\mathrm{a}$ & $1,43 \pm 0,28$ & $\mathrm{~b}$ & $* * *$ \\
\hline MR 2018-19 & $15(0,88)$ & $3,15 \pm 0,14$ & $\mathrm{a}$ & $2,00 \pm 0,13$ & $a b$ & $1,15 \pm 0,24$ & $\mathrm{~b}$ & $* * *$ \\
\hline MR 2019-20 & $15(0,83)$ & $2,86 \pm 0,16$ & $\mathrm{a}$ & $2,31 \pm 0,15$ & $\mathrm{~b}$ & $0,55 \pm 0,17$ & $\mathrm{a}$ & $* *$ \\
\hline MR 2020-21 & $15(0,83)$ & $3,17 \pm 0,10$ & $\mathrm{a}$ & $2,23 \pm 0,13$ & $\mathrm{~b}$ & $0,93 \pm 0,18$ & $\mathrm{ab}$ & $* * *$ \\
\hline \multicolumn{9}{|l|}{ Idoma } \\
\hline Castellano & $263(0,80)$ & $3,25 \pm 0,03$ & $\mathrm{a}$ & $1,93 \pm 0,03$ & $\mathrm{~b}$ & $1,32 \pm 0,05$ & $\mathrm{a}$ & $* * *$ \\
\hline Inglés & $71(0,82)$ & $3,54 \pm 0,06$ & $\mathrm{~b}$ & $1,78 \pm 0,05$ & $\mathrm{a}$ & $1,76 \pm 0,09$ & $\mathrm{~b}$ & $* * *$ \\
\hline \multicolumn{9}{|l|}{ Género } \\
\hline Femenino & $220(0,80)$ & $3,32 \pm 0,04$ & a & $1,85 \pm 0,03$ & $\mathrm{a}$ & $1,46 \pm 0,05$ & $\mathrm{a}$ & $* * *$ \\
\hline Masculino & $114(0,81)$ & $3,31 \pm 0,05$ & $\mathrm{a}$ & $1,99 \pm 0,04$ & $\mathrm{~b}$ & $1,32 \pm 0,07$ & $\mathrm{a}$ & $* * *$ \\
\hline Total & $334(0,80)$ & $3,32 \pm 0,03$ & & $1,90 \pm 0,02$ & & $1,41 \pm 0,04$ & & $* * *$ \\
\hline
\end{tabular}

${ }^{1}$ Letras diferentes en la misma columna indican diferencias significativas (P-value $\left.<0,05\right)$ entre grupos según el test de Tukey. ${ }^{2 * * *}$ : $\mathrm{P}<0,0001$ 


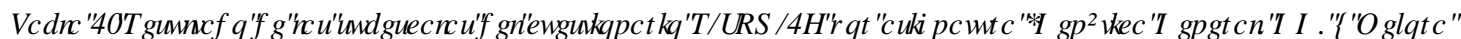

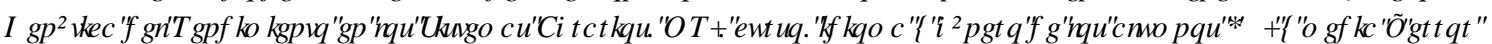

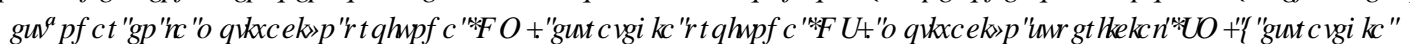
XSHIIFIDO66"ए

\begin{tabular}{|c|c|c|c|c|c|c|c|c|}
\hline & DM & & DS & & SM & & SS & \\
\hline \multicolumn{9}{|l|}{ Asignatura } \\
\hline GG & $3,40 \pm 0,03$ & $\mathrm{~b}^{1}$ & $3,32 \pm 0,04$ & $\mathrm{~b}$ & $1,50 \pm 0,03$ & $\mathrm{a}$ & $2,22 \pm 0,03$ & $\mathrm{a}$ \\
\hline MR & $3,20 \pm 0,08$ & $\mathrm{a}$ & $2,98 \pm 0,09$ & $\mathrm{a}$ & $1,83 \pm 0,08$ & $\mathrm{~b}$ & $2,38 \pm 0,09$ & $\mathrm{a}$ \\
\hline \multicolumn{9}{|l|}{ Curso } \\
\hline GG 2018-19 & $3,35 \pm 0,06$ & a & $3,24 \pm 0,07$ & $\mathrm{a}$ & $1,61 \pm 0,04$ & $\mathrm{~b}$ & $2,33 \pm 0,06$ & $\mathrm{~b}$ \\
\hline GG 2019-20 & $3,46 \pm 0,06$ & $\mathrm{a}$ & $3,28 \pm 0,07$ & $\mathrm{a}$ & $1,45 \pm 0,04$ & $\mathrm{a}$ & $2,22 \pm 0,06$ & $a b$ \\
\hline GG 2020-21 & $3,40 \pm 0,05$ & $\mathrm{a}$ & $3,41 \pm 0,06$ & $\mathrm{a}$ & $1,46 \pm 0,04$ & $\mathrm{a}$ & $2,12 \pm 0,05$ & $\mathrm{a}$ \\
\hline MR 2017-18 & $3,22 \pm 0,22$ & $\mathrm{a}$ & $3,18 \pm 0,19$ & $\mathrm{a}$ & $1,55 \pm 0,14$ & $\mathrm{a}$ & $2,00 \pm 0,20$ & $\mathrm{a}$ \\
\hline MR 2018-19 & $3,27 \pm 0,16$ & $\mathrm{a}$ & $3,04 \pm 0,16$ & $\mathrm{a}$ & $1,63 \pm 0,11$ & $\mathrm{a}$ & $2,37 \pm 0,18$ & $\mathrm{ab}$ \\
\hline MR 2019-20 & $3,01 \pm 0,17$ & $\mathrm{a}$ & $2,71 \pm 0,21$ & $\mathrm{a}$ & $2,13 \pm 0,16$ & $\mathrm{~b}$ & $2,48 \pm 0,16$ & $\mathrm{ab}$ \\
\hline MR 2020-21 & $3,29 \pm 0,11$ & $\mathrm{a}$ & $3,04 \pm 0,14$ & $\mathrm{a}$ & $1,92 \pm 0,15$ & $a b$ & $2,55 \pm 0,17$ & $\mathrm{~b}$ \\
\hline \multicolumn{9}{|l|}{ Idoma } \\
\hline Castellano & $3,35 \pm 0,04$ & $\mathrm{a}$ & $3,24 \pm 0,04$ & $\mathrm{a}$ & $1,52 \pm 0,03$ & a & $2,26 \pm 0,04$ & $\mathrm{~b}$ \\
\hline Inglés & $3,55 \pm 0,07$ & $\mathrm{~b}$ & $3,53 \pm 0,07$ & $b$ & $1,46 \pm 0,05$ & $\mathrm{a}$ & $2,10 \pm 0,06$ & a \\
\hline \multicolumn{9}{|l|}{ Género } \\
\hline Femenino & $3,38 \pm 0,04$ & $\mathrm{a}$ & $3,26 \pm 0,04$ & $\mathrm{a}$ & $1,49 \pm 0,03$ & $\mathrm{a}$ & $2,22 \pm 0,04$ & $\mathrm{a}$ \\
\hline Masculino & $3,36 \pm 0,05$ & $\mathrm{a}$ & $3,26 \pm 0,06$ & $\mathrm{a}$ & $1,68 \pm 0,04$ & $\mathrm{~b}$ & $2,30 \pm 0,05$ & $\mathrm{a}$ \\
\hline Total & $3,37 \pm 0,03$ & & $3,26 \pm 0,04$ & & $1,56 \pm 0,02$ & & $2,24 \pm 0,03$ & \\
\hline
\end{tabular}

${ }^{1}$ Letras diferentes en la misma columna indican diferencias significativas ( $\mathrm{P}$-value $\left.<0,05\right)$ entre grupos según el test de Tukey.

La distribución del alumnado en función de su puntuación en DA y SA se muestra en la figura 1. Tres veces más alumnos/as mostraron un DA mayor que la media y SA menor que la media comparado con el comportamiento opuesto, seguidos por comportamientos intermedios, lo que parece indicar una alta implicación del alumnado. Resultados similares se obtuvieron en otros estudios (Jeong HWDO, 2019; LeivaBrondo, Cebolla-Cornejo, Peiró, Andrés-Colas, HWDO, 2020). 


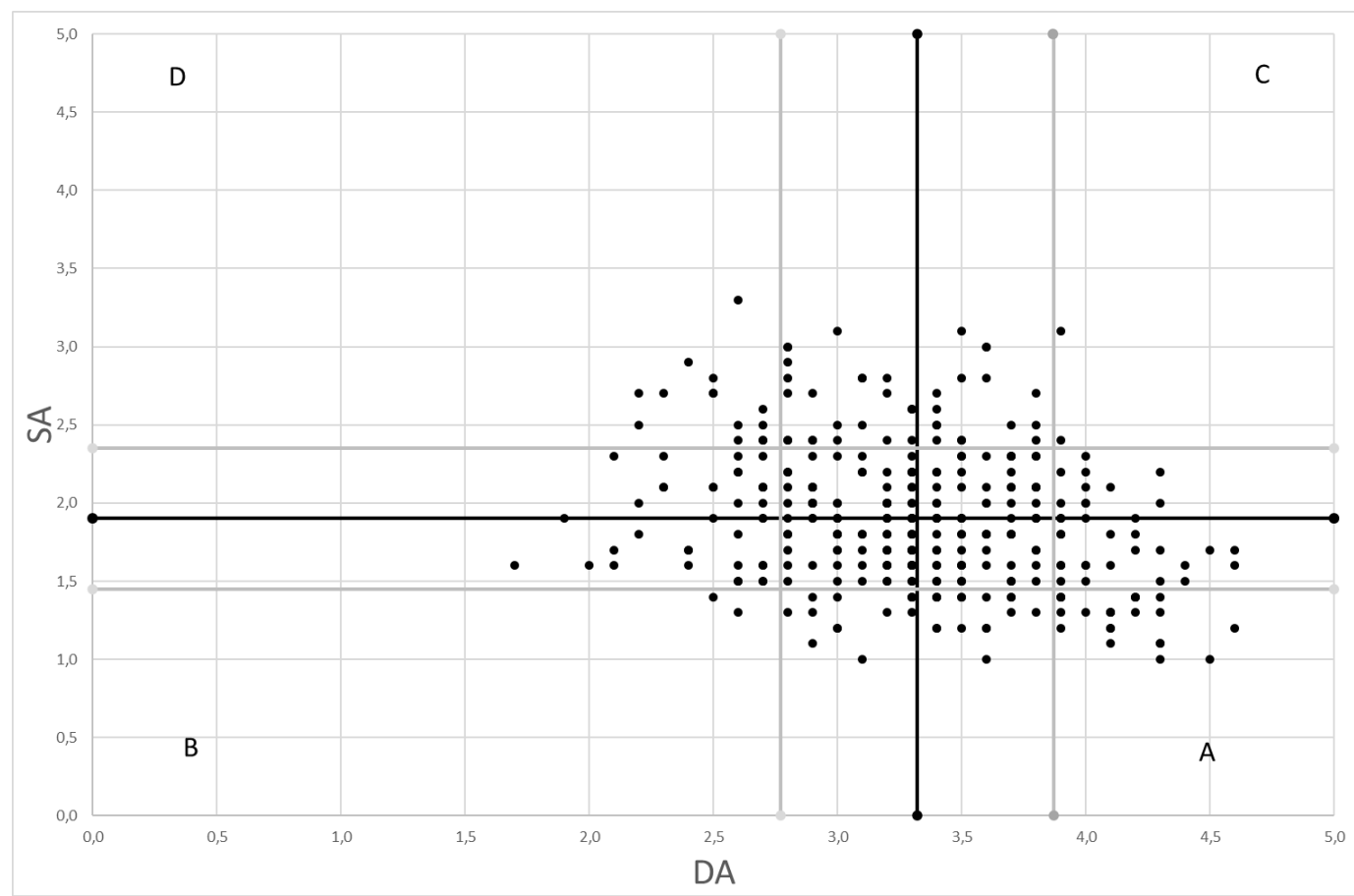

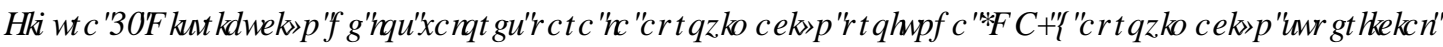

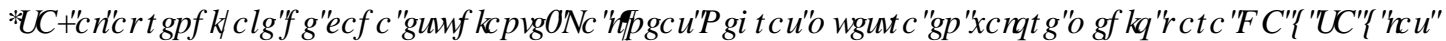

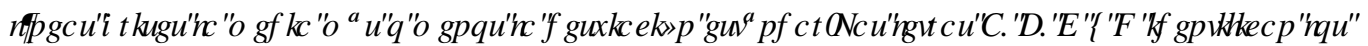 FXDOUQUAVGHDJ IIIFR]}

De forma similar a lo descrito en otros estudios, se observaron altas correlaciones entre la escala principal y sus subescalas relacionadas (Tabla 3), así como correlaciones negativas entre las escalas y subescalas opuestas (Biggs, Kember and Leung, 2001; Immekus and Imbrie, 2010; Socha and Sigler, 2014; LeivaBrondo, Cebolla-Cornejo, Peiró, Andrés-Colas, $H W D$, 2020). Estos resultados apoyan la existencia de dos escalas principales. La estructura interna del cuestionaria ha sido estudiada en distintos contextos académicos y culturales y la mayoría de los estudios avalan la existencia de dos enfoques principales profundo y superficial (Justicia $H W D O$, 2008; Immekus and Imbrie, 2010; Socha and Sigler, 2014; Xie, 2014; Zakariya $H W D O, 2020$ ), aunque también se puede encontrar un enfoque estratégico (Tait, Entwistle and McCune, 1998; Entwistle and Hilary, 2013).

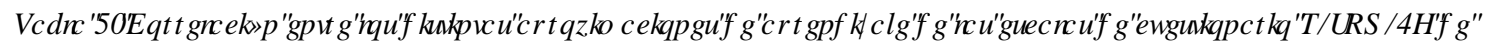

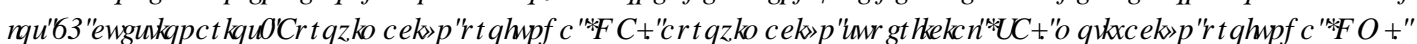

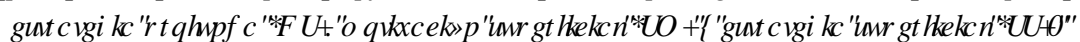

\begin{tabular}{cccccc}
\hline \multicolumn{7}{c}{$\mathbf{D A}$} & $\mathbf{S A}$ & $\mathbf{D M}$ & $\mathbf{D S}$ & $\mathbf{S M}$ \\
\hline $\mathbf{S A}$ & $-0,29 * * *$ & & & \\
$\mathbf{D M}$ & $0,87 * * *$ & $-0,22 * * *$ & & & \\
$\mathbf{D S}$ & $0,90 * * *$ & $-0,28 * * *$ & $0,57 * * *$ & & \\
$\mathbf{S M}$ & $-0,21 * * *$ & $0,84 * * *$ & $-0,18 * * *$ & $-0,20 * * *$ & \\
$\mathbf{S S}$ & $-0,28 * * *$ & $0,91 * * *$ & $-0,21 * * *$ & $-0,29 * * *$ & $0,55 * * *$ \\
\hline \multicolumn{7}{c}{$* * *: \mathrm{P}<0,001$} &
\end{tabular}




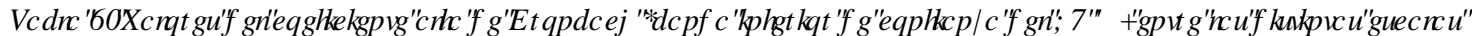

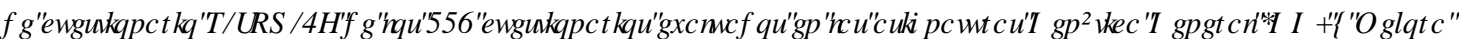

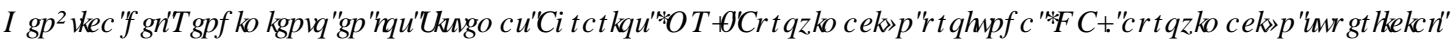

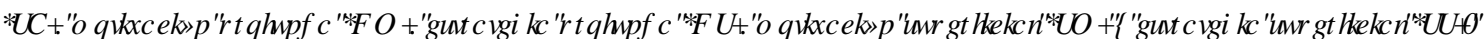

\begin{tabular}{ccccccc}
\hline \multicolumn{1}{c}{ DA } & SA & DM & DS & SM & SS \\
\hline Asignatura & & & & & \\
GG & $0,75(0,71)$ & $0,65(0,60)$ & $0,53(0,46)$ & $0,67(0,63)$ & $0,41(0,33)$ & $0,49(0,41)$ \\
MR & $0,76(0,72)$ & $0,72(0,68)$ & $0,62(0,56)$ & $0,68(0,63)$ & $0,61(0,55)$ & $0,56(0,49)$ \\
\hline Curso & & & & & & \\
GG 2018-19 & $0,73(0,68)$ & $0,67(0,60)$ & $0,56(0,47)$ & $0,62(0,54)$ & $0,41(0,29)$ & $0,55(0,48)$ \\
GG 2019-20 & $0,79(0,76)$ & $0,64(0,59)$ & $0,62(0,56)$ & $0,71(0,66)$ & $0,71(0,32)$ & $0,47(0,39)$ \\
GG 2020-21 & $0,72(0,68)$ & $0,62(0,57)$ & $0,39(0,30)$ & $0,67(0,62)$ & $0,37(0,28)$ & $0,44(0,35)$ \\
& & & & & \\
MR 2017-18 & $0,87(0,85)$ & $0,75(0,70)$ & $0,82(0,79)$ & $0,70(0,64)$ & $0,67(0,60)$ & $0,67(0,60)$ \\
MR 2018-19 & $0,73(0,67)$ & $0,69(0,63)$ & $0,58(0,50)$ & $0,62(0,54)$ & $0,52(0,42)$ & $0,55(0,49)$ \\
MR 2019-20 & $0,80(0,78)$ & $0,73(0,69)$ & $0,68(0,63)$ & $0,80(0,77)$ & $0,52(0,44)$ & $0,54(0,47)$ \\
MR 2020-21 & $0,42(0,34)$ & $0,64(0,59)$ & $0,25(0,14)$ & $0,40(0,31)$ & $0,55(0,49)$ & $0,51(0,44)$ \\
\hline Idoma & & & & & & \\
Castellano & $0,74(0,70)$ & $0,70(0,68)$ & $0,68(0,63)$ & $0,63(0,53)$ & $0,53(0,45)$ & $0,45(0,66)$ \\
Inglés & $0,76(0,73)$ & $0,73(0,65)$ & $0,65(0,61)$ & $0,61(0,61)$ & $0,61(0,55)$ & $0,55(0,69)$ \\
\hline Género & & & & & & \\
Femenino & $0,77(0,74)$ & $0,68(0,64)$ & $0,60(0,55)$ & $0,66(0,61)$ & $0,51(0,44)$ & $0,50(0,42)$ \\
Masculino & $0,73(0,69)$ & $0,66(0,61)$ & $0,44(0,36)$ & $0,71(0,66)$ & $0,45(0,37)$ & $0,52(0,45)$ \\
\hline Total & $0,75(0,72)$ & $0,68(0,63)$ & $0,55(0,48)$ & $0,68(0,63)$ & $0,50(0,43)$ & $0,50(0,43)$ \\
\hline
\end{tabular}

El alfa de Cronbach se utilizó para evaluar la fiabilidad interna del cuestionario. Se obtuvieron valores superiores a 0,7 para las escalas principales, lo que indica una alto nivel de ajuste (Hundleby and Nunnally, 2006). El modelo ha sido probado con distintas asociaciones de factores y un modelo con dos factores de primer orden da un buen ajuste (Justicia HWDO, 2008; Fryer HWDO, 2012; Socha and Sigler, 2014; Vaughan, 2018; Zakariya, 2019; Zakariya $H M D O$, 2020), aunque en algunos casos se ha sugerido la eliminación de algunas preguntas (Immekus and Imbrie, 2010; Vaughan, 2016; Zakariya, 2019).

En general, los resultados mostraron un elevado enfoque profundo y mayor que el enfoque superficial en ambas asignaturas, sugiriendo un alto grado de implicación y una motivación intrínseca del alumnado. Diversos factores pueden explicar este enfoque, que se ve influido por factores personales, contextuales y percibidos (Biggs, 1987; Baeten $H W D O$, 2010). En nuestro caso la elección de los estudios está altamente motivada, debido a la alta nota de admisión en el caso del grado y a la alta especificidad de los estudios en el caso del máster. En cualquier caso, se observó un menor enfoque de aprendizaje en los estudios de máster. Este hecho ya se ha observado en otros estudios (Zeegers, 2001; Leiva-Brondo, Cebolla-Cornejo, Peiró, Andrés-Colas, $H W D O, 2020)$ y se podría explicar porque el alumnado opta por un enfoque más estratégico, lo que disminuye su enfoque profundo, pero sin llegar a ser superficial. La metodología que se utiliza en la asignatura también es un factor que influye en el enfoque de aprendizaje (Biggs, 1987; Eley, 1992; Zeegers, 2001) y estudios premilimares muestran que en estas asignaturas no modifican el elevado enfoque profundo inicial (Leiva-Brondo, Cebolla-Cornejo, Peiró and Pérez-de-Castro, 2020; Leiva-Brondo HWDO, 2021). 


\section{Conclusiones}

El enfoque de aprendizaje es una elección del alumnado que depende de factores personales, contextuales y situacionales. En las dos asignaturas evaluadas en el presente estudio según el cuestionario R-SPQ-2F los estudiantes muestran un elevado enfoque de aprendizaje profundo, aunque mayor en grado que en máster, indicando un interés intrínseco en aprender más que en superar la asignatura. El idioma de impartición es un factor que influye, pero otros factores pueden explicar esta diferencia. El género y el curso académico no afectaron al enfoque de aprendizaje. La edad y la experiencia universataria del estudiante pueden ser factores que hagan que opte por un enfoque menos profundo, aunque no más supercial. Es posible que un enfoque más estratégico pueda explicar estos resultados. Más estudios en otras asignaturas y contextos académicos son necesarios para corroborar los resultados aquí presentados.

\section{Agradecimientos}

La publicación de este trabajo ha sido parcialmente financiada por un proyecto de innovación educativa (PIME/19-20/168) concedido por el Vicerrectorado de Estudios, Calidad y Acreditación de la Universitat Politècncia de València (UPV). También los autores agradecen el apoyo dado por el Instituto de Ciencias de la Educación (ICE) de la UPV.

\section{Referencias}

Baeten, M. $H M D O$ (2010) 'Using student-centred learning environments to stimulate deep approaches to learning: Factors encouraging or discouraging their effectiveness', ( GXFDURQDO5 HMDDFKI5 HIILZ. Elsevier Ltd, 5(3), pp. 243-260. doi: 10.1016/j.edurev.2010.06.001.

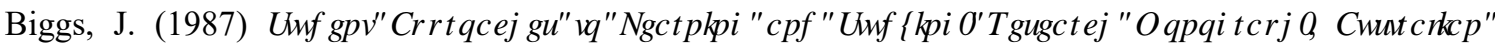
( GXFDURQ5 HMDUFKDQG' HHHRSP HQW Melbourne: Australian Council Educational Research (ACER). Available at: https://eric.ed.gov/?id=ED308201.

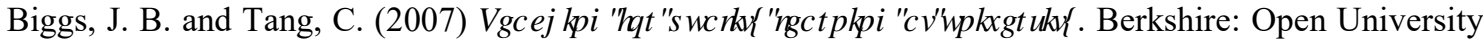
Press/McGraw-Hill Education.

Biggs, J., Kember, D. and Leung, D. Y. P. (2001) 'The revised two-factor Study Process Questionnaire: RSPQ-2F', 7KH \%ULM NRXLQDO RI HEXFDURQDO SKFKRQRJ, 71(1), pp. 133-149. doi: 10.1348/000709901158433.

Bobe, B. J. and Cooper, B. J. (2017) 'The effect of language proficiency on approaches to learning and satisfaction of undergraduate accounting students', \$FFRQMQJ ( GXFDMRQ Taylor \& Francis, 28(2), pp. 149-171. doi: 10.1080/09639284.2017.1396481.

Dong, N. HWDO (2019) 'Approaches to learning IFRS by Chinese accounting students', -RXLDORI \$FFRQMQJLC GXFDMRQ. Elsevier Ltd, 48, pp. 1-11. doi: 10.1016/j.jaccedu.2019.04.002.

Eley, M. G. (1992) 'Differential adoption of study approaches within individual students', +LKKU ( GXFDMRQ 23(3), pp. 231-254. doi: 10.1007/BF00145015.

Emilia, O., Bloomfield, L. and Rotem, A. (2012) 'Measuring students' approaches to learning in different clinical rotations', \%0 \&10 HGFDO GXFDURQ 12(1), p. 114. doi: 10.1186/1472-6920-12-114.

Entwistle, N. and Entwistle, D. (2003) 'Preparing for examinations: The interplay of memorising and understanding, and the development of knowledge objects', + IJKHU GXFDURQ5 HMDFKDQQF' HMHRSP HQW, 22(1), pp. 19-41. doi: 10.1080/0729436032000056562.

Entwistle, N. and Hilary, T. (2013) 'Approaches and Study Skills Inventory for Students (ASSIST) (incorporating the Revised Approaches to Studying Inventory - RASI)'. Available at: https://www.researchgate.net/publication/260291730_Approaches_and_Study_Skills_Inventory_for_Stud ents_ASSIST_incorporating_the_Revised_Approaches_to_Studying_Inventory_-_RASI.

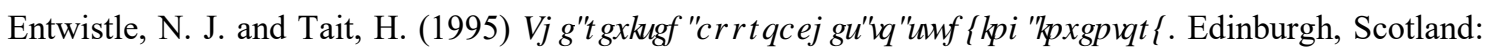
Centre for Research on Learning and Instruction. 


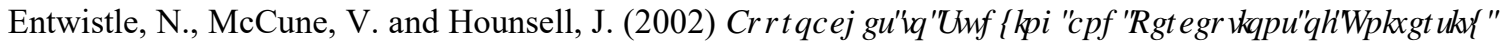

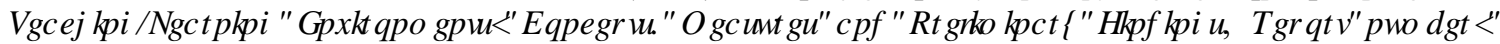

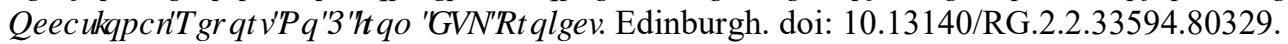

Frăsineanu, E. S. (2013) 'Approach to Learning Process: Superficial Learning and Deep Learning at Students', 3 URFHDDI6RFWODQG\%/KDVIRLDOGFIHQFH, 76, pp. 346-350. doi: 10.1016/j.sbspro.2013.04.125.

Fryer, L. K. $H W D O$ (2012) 'The adaptation and validation of the CEQ and the R-SPQ-2F to the Japanese tertiary environment', \%UWK-RXLDORIL( GXFDURQDOB K FKR(RJ।, 82(4), pp. 549-563. doi: 10.1111/j.20448279.2011.02045.x.

Gijbels, D. $H W D O(2005)$ 'The relationship between students' approaches to learning and the assessment of learning outcomes', ( XLRSHDQ -RXLQDO RI 3 XFKRRJII RI ( GXFDMRQ 20(4), pp. 327-341. doi: 10.1007/BF03173560.

Gow, L. and Kember, D. (1990) 'Does higher education promote independent learning?', +LKH】 ( GXFDURQ 19(3), pp. 307-322. doi: 10.1007/BF00133895.

Graham, N. C., Entwistle, N. and Ramsden, P. (1984) 'Understanding Student Learning', \% ЖUKK-RХLDO RIC GXFDURQDO6WGHN, 32(3), p. 284. doi: 10.2307/3121589.

Hundleby, J. D. and Nunnally, J. (2006) 3 K FKRP HMFI7KHU, \$P HUFDQ( GXFDURQDO5 HMDLFK-RXLQDO Edited by McGraw-Hil. New York, USA. doi: 10.2307/1161962.

Immekus, J. C. and Imbrie, P. K. (2010) 'A Test and Cross-Validation of the Revised Two-Factor Study Process Questionnaire Factor Structure Among Western University Students', ( GXFDURQDO DQG 3 K FKRRJIFDOO HDXUP HQW70(3), pp. 495-510. doi: 10.1177/0013164409355685.

Jeong, J. S. HMDO (2019) 'Examination of students' engagement with R-SPQ- 2F of learning approach in flipped sustainable science course', - RXLQDORIDOOWFI6FIHQFH( GXFDURQ 18(6), pp. 880-891. Available at: http://www.scientiasocialis.lt/jbse/?q=node/811.

Jiyed, O. HMDO (2020) 'The Revised Two-Factor Study process questionnaire ( R-SPQ-2F ): Translation and Confirmatory Factor examination among the Moroccan higher education context . The R-SPQ-2F history and relevance. The study objective . , $6<1: \$ 1,164(3)$, pp. 240-250.

Justicia, F. $H W D O(2008)$ 'The Revised Two-Factor Study Process Questionnaire (R-SPQ-2F): Exploratory

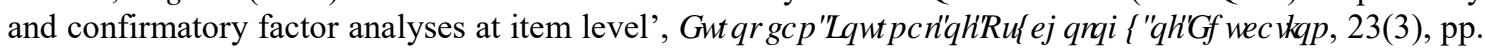
355-372. doi: 10.1007/BF03173004.

Leiva-Brondo, M. HWDO (2018a) 'DEEP LEARNING APPROACH FOR STUDENTS OF PLANT BREEDING IN A MASTER DEGREE', in Gómez Chova, L., López Martínez, A., and Candel Torres, I.

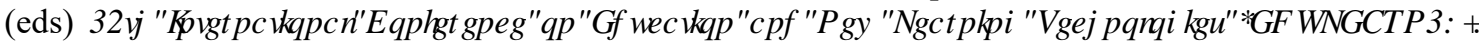
Palma, Mallorca: International Association of Technology, Education and Development. IATED, pp. 3437 3442. doi: 10.21125/edulearn.2018.0890.

Leiva-Brondo, M. HWDO (2018b) 'FIRST YEAR LIFE SCIENCE STUDENTS DEEP LEARNING APPROACH: A PRELIMINARY REPORT', in Gómez Chova, L., López Martínez, A., and Candel Torres,

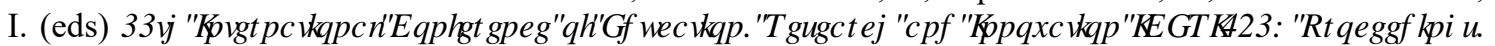
Sevilla (Spain): International Association of Technology, Education and Development. IATED, pp. 542548. doi: 10.21125/iceri.2018.1111.

Leiva-Brondo, M. $H W D O(2019)$ 'Evaluación de la aproximación al aprendizaje de estudiantes de ciencias

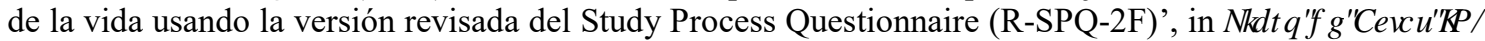

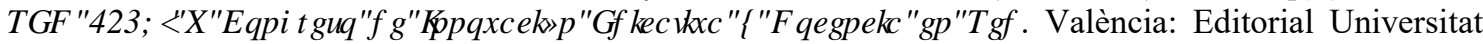
Politècnica de València, pp. 156-170. doi: 10.4995/INRED2019.2019.10406.

Leiva-Brondo, M., Cebolla-Cornejo, J., Peiró, R. and Pérez-de-Castro, A. M. (2020) 'COMPARISON STUDENT LEARNING APPROACH OF FIRST YEAR LIFE SCIENCE STUDENTS', in TI UKDQQXDO , QULQDURQDO\&RQHHHQH RQ ( GXFDURQDQG $1 \mathrm{HZ}$ / HDQQQJ 7 7HKQRRJIH. International Association of Technology, Education and Development. IATED, pp. 2019-2026. doi: 10.21125/edulearn.2020.0638. 
Leiva-Brondo, M., Cebolla-Cornejo, J., Peiró, R., Andrés-Colas, N., HWDO (2020) 'Study Approaches of Life Science Students Using the Revised Two-Factor Study Process Questionnaire (R-SPQ-2F)', ( GXFDRRQ 6FHQFH, 10(7), p. 173. doi: 10.3390/educsci10070173.

Leiva-Brondo, M. HWDO (2021) 'STUDENT APPROACH TO LEARNING IN A DEGREE LEVEL SUBJECT: EVOLUTION OVER THE TERM', in IT UK, QUHQDURQDO 7HFKQRQRJI ( GXFDURQ DQG

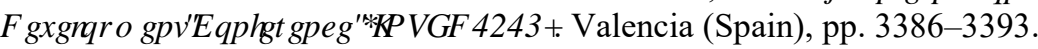

Leung, D. Y. P., Ginns, P. and Kember, D. (2008) 'Examining the Cultural Specificity of Approaches To Learning in Universities in Hong Kong and Sydney', -RXLQDORI \&LRW \&XQKUDO3 V FKRRJ।, 39(3), pp. 251-266. doi: 10.1177/0022022107313905.

López-Aguado, M. and Gutiérrez-Provecho, L. (2018) 'Checking the underlying structure of R-SPQ-2F using covariance structure analysis', \&XQXWD I ( GXFDFIRQ. Routledge, 30(1), pp. 105-141. doi: 10.1080/11356405.2017.1416787.

Marton, F. and Säljö, R. (1976a) 'On qualitative differences in learning-II outcome as a function of the learners's conception of the task', \%ULMKL-RXLQDORID GXFDURQDO3 W FKRQRJ।, 46(2), pp. 115-127. doi: 10.1111/j.2044-8279.1976.tb02304.x.

Marton, F. and Säljö, R. (1976b) 'On qualitative differences in learning: I-Outcome and process', \% - RXLQDORIL GXFDURQDOB V FKRRJ। , 46(1), pp. 4-11. doi: 10.1111/j.2044-8279.1976.tb02980.x.

May, W. $H M D O$ (2012) 'The relationship between medical students' learning approaches and performance on a summative high-stakes clinical performance examination', 0 HAFDO7HDKHU 34(4), pp. e236-e241. doi: 10.3109/0142159X.2012.652995.

Merino Soto, C. and Kumar Pradhan, R. (2013) 'Validación estructural del R-SPQ-2F: un análisis factorial confirmatorio', 5HLWD ' IJLDO GH ,QYHWDFIYQ HQ ' RFHQFD 8 QIVHWWUD, (1), p. 111. doi: 10.19083/ridu.7.190.

Michael, W. B., Michael, J. J. and Zimmerman, W. S. (1985) 6WG \\$ UWXGHDQQGO HKRGV6XUHН San Diego, CA: Educational and Industrial Testing Service.

Mogre, V. and Amalba, A. (2014) 'Assessing the reliability and validity of the Revised Two Factor Study Process Questionnaire (RSPQ2F) in Ghanaian medical students', - RXLQDORIL( GXFDMRQDOC YDODURQIRU + HDOKL3 URIHMRQV, 11, p. 19. doi: 10.3352/jeehp.2014.11.19.

Muñoz San Roque, I., Prieto Navarro, L. and Torre Puente, J. C. (2012) 'Enfoques de aprendizaje, autorregulación, autoeficacia, competencias y evaluación. Un estudio descriptivo de estudiantes de

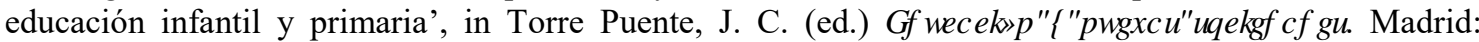
Universidad Pontificia Comillas, pp. 237-266.

Richardson, J. T. E. (2013) 'Approaches to studying across the adult life span: Evidence from distance education', / HDQQQJ] DQGT ,QAYIGXDO ' IIHHQFH. Elsevier Inc., 26, pp. 74-80. doi: 10.1016/j.lindif.2013.04.012.

Richardson, J. T. E. and King, E. (1998) 'Adult Students in Higher Education: Burden or Boon?', 7KH - RXLQDORI+ LKHU( GXFDURQ 69(1), p. 65. doi: 10.2307/2649182.

Rubin, M. $H W D O(2018)$ 'Older Women, Deeper Learning, and Greater Satisfaction at University: Age and Gender Predict University Students' Learning Approach and Degree Satisfaction', - RXLQDORI' LYHUMLQ + IJKHU( GXFDURQ 11(1), pp. 82-96. doi: 10.1037/dhe0000042.

Salamonson, Y. $H M D O$ (2013) 'Learning approaches as predictors of academic performance in first year health and science students', 1 XUH ( GXFDMRQ 7RGD. Elsevier Ltd, 33(7), pp. 729-733. doi: 10.1016/j.nedt.2013.01.013.

Schmeck, R. R., Ribich, F. and Ramanaiah, N. (1977) 'Development of a Self-Report Inventory for Assessing Individual Differences in Learning Processes', \$SSGHCA3 V FKRRJIFDOO HDXUP HQW1(3), pp. 413-431. doi: 10.1177/014662167700100310.

Severiens, S. and Dam, G. (1998) 'A multilevel meta-analysis of gender differences in learning

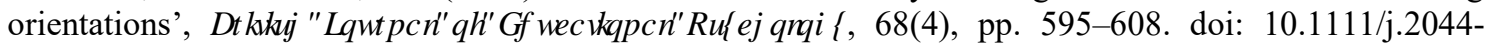
8279.1998.tb01315.x. 
Shaik, S. A. $H W D O(2017)$ 'Assessing Saudi medical students learning approach using the revised two-factor study process questionnaire', , QULQDURQDO - RXLQDO RID 0 HIFDO ( GXFDMRQ 8, pp. 292-296. doi: 10.5116/ijme.5974.7a06.

Socha, A. and Sigler, E. A. (2014) 'Exploring and "reconciling" the factor structure for the Revised Twofactor Study Process Questionnaire', / HDQQJDDQG, QCVIGXDO IIIHHQFH. Elsevier Inc., 31, pp. 43-50. doi: 10.1016/j.lindif.2013.12.010.

Stes, A., de Maeyer, S. and Van Petegem, P. (2013) 'Examining the Cross-Cultural Sensitivity of the Revised Two-Factor Study Process Questionnaire (R-SPQ-2F) and Validation of a Dutch Version', 3/ R6ロ 21 ( . Edited by O. García, 8(1), p. e54099. doi: 10.1371/journal.pone.0054099.

Tait, H., Entwistle, N. J. and McCune, V. (1998) 'ASSIST: a reconceptualisation of the Approaches to Studying Inventory', in Rust, C. (ed.) , P SLRYIJ $\square$ WGHQWDVCODQHWV Oxford: Oxford Brookes University, The Oxford Centre for Staff and Learning Development., pp. 262-271.

Vaughan, B. (2016) 'Confirmatory factor analysis of the Study Process Questionnaire in an Australian osteopathy student population', , QUHQDURQDO- RXLQDORIL2 WHRSDWKIFIO HOFFQH Elsevier Ltd, 20, pp. 6267. doi: 10.1016/j.ijosm.2016.03.001.

Vaughan, B. (2018) 'A Rasch analysis of the Revised Study Process Questionnaire in an Australian osteopathy student cohort', 6WGHWIQ( GXFDURQDOC YDODWRQ. Elsevier, 56(December), pp. 144-153. doi: 10.1016/j.stueduc.2017.12.003.

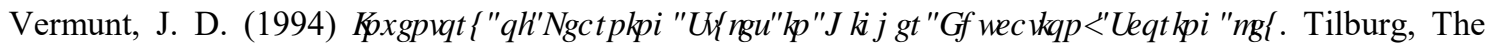
Netherlands: Tilburg University, Department of Educational Psychology.

Weinstein, C. E. (1987) / HDQQDШDQG66WG [6WDWJ IHM, QYHQURU. Clearwater, FL: H \& H Publishing.

Xie, Q. (2014) 'Validating the Revised Two-Factor Study Process Questionnaire among Chinese University Students', 7KH, QULQDURQDO RXLQDORIL( GXFDURQDODQGF3 V FKRRJ IFDO16(1), pp. 4-19.

Zakariya, Y. F. (2019) 'Study approaches in higher education mathematics: Investigating the statistical behaviour of an instrument translated into norwegian', (GXFDMRQ 6FIHQFH, 9(3). doi: 10.3390/educsci9030191.

Zakariya, Y. F. $H W D O(2020)$ 'University students' learning approaches: An adaptation of the revised twofactor study process questionnaire to Norwegian', 6WGHWIQ( GXFDURQDO( YDODURQ. Elsevier, 64(May 2019), p. 100816. doi: 10.1016/j.stueduc.2019.100816.

Zeegers, P. (2001) 'Approaches to learning in science: A longitudinal study', \%UMKL RXLQDORIL GXFDMRQDO 3 KFKRRJ।, 71(1), pp. 115-132. doi: 10.1348/000709901158424. 